\title{
NÍVEIS DE ATIVIDADE FÍSICA EM DIFERENTES MODALIDADES ESPORTIVAS: UM PROGRAMA NÃO CONVENCIONAL DE EDUCA- ÇÃO FÍSICA ESCOLAR
}

\author{
Rodrigo B. Moreira \\ Universidade Luterana do Brasil, São Jerônimo, Rio Grande do Sul, Brasil
}

Giovani L. D. Nina

Universidade Luterana do Brasil, São Jerônimo, Rio Grande do Sul, Brasil

Anelise R. Gaya

Universidade Federal do Rio Grande do Sul, Porto Alegre, Rio Grande do Sul, Brasil

Pablo L.D. Nina

Instituto Estadual de Educação Vasconcelos Jardim, General Câmara, Rio Grande do Sul, Brasil

Adriana T. de Lemos

Universidade Federal de Ciências da Saúde de Porto Alegre, Porto Alegre, Rio Grande do Sul, Brasil

\section{Adroaldo Gaya}

Universidade Federal do Rio Grande do Sul, Porto Alegre, Rio Grande do Sul, Brasil

\begin{abstract}
Resumo
Objetivos: a) avaliar a intensidade, tempo de deslocamento ativo e gasto energético (kcal), em diferentes modalidades esportivas, estratificado por sexo; b) conforme quantidade de modalidades esportivas frequentadas durante a semana na escola, identificar a contribuição da educação física escolar quanto ao tempo AFMV realizada durante a semana. Métodos: Estudo de caso: programa de educação física do Instituto Estadual de Educação Vasconcelos Jardim no município de General Câmara/RS. Amostra do tipo aleatória estratificada por sexo e faixa etária. Foram selecionados 206 escolares, 112 do sexo masculino e 94 do sexo feminino. Os escolares foram avaliados em 72 aulas por GPS da marca GARMIN, modelo 310XT, com frequencímetro. A intensidade da AF foi categorizada conforme critérios sugeridos por Armostrong (1998): Para tratamento dos dados utilizou-se: (1) estatística descritiva identificando a proporção de tempo em $\mathrm{AF}$ nos diferentes níveis de intensidade e, médias e desvios-padrão para a descrição dos dados; (2) estatística inferencial foi usado o teste " $t$ " de student para comparação entre os sexos; e ANOVA com post hoc de Bonferroni para comparação entre as categorias de idades, todos assumindo previamente o nível de significância de 5\%. Resultados: Quanto ao tempo semanal engajado em práticas de AF realizadas de forma moderada a vigorosa, observamos que o somatório de AFMV praticadas nas modalidades na escola demonstram resultados significativos, somente com as aulas de educação física os rapazes cumprem com cerca de 2/3 da carga horária semanal recomendada e, as moças com valores inferiores ao apresentado pelos rapazes, cumprem com cerca de $50 \%$ da carga horária sugerida. Consideramos estes resultados o diferencial desta proposta, neste formato os escolares ampliam o tempo de prática de AF na escola contribuindo de forma significativa quando comparada com as recomendações de 300 minutos de AF moderada a vigorosa por semana.
\end{abstract}

Palavras-chave: Atividade Física. Intensidade e duração. Escolares.

\section{Introdução}


A Organização Mundial da Saúde ${ }^{1}$ recomenda para crianças e adolescentes com idades entre 5 e 17 anos, a prática de AF nos diferentes domínios (lazer, deslocamento, serviços domésticos, trabalho e atividades físicas na escola ou fora dela) de no mínimo 60 minutos diários ou, pelo menos, 300 minutos por semana, com intensidades de moderada a vigorosa, como instrumento de prevenção de doenças e promoção da saúde.

Ainda que tenhamos visto constantes melhorias nas condições de saúde e educação na última década, alguns aspectos negativos acompanharam este processo, os hábitos alimentares inadequados, o uso de cigarros, álcool e drogas, bem como a inatividade física acompanhada ao incremento do tempo em frente à televisão, à internet e celulares, a diminuição no lazer ativo e a redução do número de aulas (períodos semanais) de educação física nas escolas, têm sido observadas e têm causado implicações no desenvolvimento corporal de nossos jovens ${ }^{2,3}$.

Perante este cenário, especialistas ${ }^{4-7}$ têm sugerido programas de promoção da AF como estratégia para conter o avanço na incidência de doenças crônicas não transmissíveis. Enfim, se faz necessário, constituir intervenções eficazes com a expectativa de melhorar comportamentos dos hábitos de vida de escolares ${ }^{8}$.

Sugere-se que a escola, de maneira geral, e a disciplina de educação física em particular, assumam a incumbência de desenvolver programas que levem os educandos a perceberem a importância de se adotar um estilo de vida saudável, fazendo com que a atividade física direcionada à promoção da saúde torne-se componente habitual no cotidiano dos escolares?.

Como agente da educação voltada para a promoção da saúde, a educação física escolar deve assumir seu papel, qual seja, a potencialidade para proporcionar situações que contemplem valores e significados para a prática de atividades físicas ${ }^{10}$. No momento atual, para a grande parte dos nossos jovens, a educação física escolar passou a ser o único momento em que realizam atividades físicas organizadas (exercícios físicos). Não obstante, é necessário construirmos uma proposta pedagógica que contemple significados e sentido na perspectiva de incentivar e promover hábitos saudáveis e um estilo de vida ativo em nossos jovens.

Desta forma, se faz premente a necessidade de avaliar os níveis de atividade física da população jovem, a fim de melhor compreender aspectos importantes dos seus hábitos, de forma a planejar intervenções e estratégias na prevenção de futuras doenças associadas ao sedentarismo, principalmente no contexto escolar.

Nesta perspectiva, a partir de uma proposta pedagógica para a educação física escolar centrada na realização de atividades esportivas, foram concebidos os seguintes objetivos: a) Avaliar a intensidade, tempo de deslocamento ativo e gasto energético (kcal), estratificado por sexo; b) Conforme quantidade de modalidades esportivas frequentadas durante a semana na escola, identificar a contribuição da educação física escolar quanto ao tempo de Atividade Física Moderada e Vigorosa (AFMV) realizada durante a semana ( $>300$ minutos por semana).

\section{Procedimentos metodológicos}

\section{Identificando a unidade de análise (o caso)}

Trata-se de um estudo de caso: o projeto alternativo de educação física escolar do Instituto Estadual de Educação Vasconcelos Jardim, do município de General Câmara/RS. O projeto de educação física nesta escola apresenta alguns pressupostos pedagógicos e algumas alternativas didáticas distintas do padrão usual das aulas de educação física. 


\section{Principais características da proposta de educação física escolar da Escola Vasconcelos Jardim}

Os objetivos:

Proporcionar aos alunos do ensino fundamental (finais) e médio, o oferecimento de uma prática pedagógica alternativa tendo em vista: a) oportunizar a ampliação do mundo motor/esportivo; b) propiciar a autonomia para tornarem-se independente e responsáveis em suas ações relacionadas a uma vida ativa e saudável; c) estimular uma vida ativa no período escolar e no futuro.

\section{A constituição das turmas:}

As turmas são constituídas por modalidades esportivas. No período letivo de 2013 foram oferecidas turmas de: Atletismo, Caminhada orientada, Mountain Bike, Futebol de campo, Futsal, Handebol e Voleibol.

Os alunos têm liberdade para escolherem quantas e quais modalidades esportivas (turmas) querem frequentar no ano letivo, sendo obrigatório, pelo menos duas modalidades por trimestre. Cada modalidade realiza-se em dois períodos semanais, totalizando para cada aluno no mínimo quatro períodos (200 minutos) obrigatórios de educação física por semana. Todavia, o aluno pode frequentar outras modalidades se assim o desejar desde que seja formalmente autorizado pelos responsáveis e, de acordo com a disponibilidade de horários. As aulas de educação física são realizadas no contra turno da escola.

As turmas são compostas e estratificadas a partir de três faixas etárias (categorias), com os seguintes objetivos gerais (1) C1 - Categoria mirim de 11 a 13 anos. Objetivos: diversidade nas práticas corporais, iniciação, ampliação dos aspetos motores e esportivos; b) C2 Categoria infantil de 14 a 15 anos. Objetivos: exploração e fixação das práticas corporais, especialização inicial, vivências e experiências motoras e esportivas; e c) C3 - Categoria juvenil de 16 a 17 anos. Objetivos: Aperfeiçoamento das práticas corporais, especialização avançada e práticas de atividades físicas na promoção da saúde.

Os planos de aula

Os planos das aulas foram planejados em Conselho de Classe e firmados pelos cinco professores de Educação Física da escola. As aulas são estruturadas em quatro partes conforme ilustrado na tabela 1 .

Tabela 1. Estrutura de aulas de Educação Física.

\begin{tabular}{|c|c|c|}
\hline Partes da aula & Atividades & Duração \\
\hline $\begin{array}{l}\text { Parte inicial: } \\
\text { Aquecimento }\end{array}$ & $\begin{array}{l}\text { Composta por atividades de preparação e aque- } \\
\text { cimento, nesta etapa são realizadas atividades } \\
\text { de alongamentos, corridas alternadas, saltos e } \\
\text { jogos recreativos com a finalidade de preparar } \\
\text { os alunos para a segunda parte da aula. }\end{array}$ & \pm 5 minutos. \\
\hline $\begin{array}{l}\text { Parte principal: } \\
\text { Formação corporal }\end{array}$ & $\begin{array}{l}\text { Composta por conteúdos relacionados com as } \\
\text { preocupações com o aprimoramento dos fun- } \\
\text { damentos e gestos técnicos da modalidade es- } \\
\text { portiva: exercícios de formação esportiva; de- } \\
\text { senvolvimento das habilidades motoras; preo- } \\
\text { cupação com as capacidades físicas. }\end{array}$ & \pm 15 minutos. \\
\hline
\end{tabular}

Parte principal: Composta por conteúdos de ensino das ativiFormação esportiva dades esportivas: preparação técnica e tática \pm 25 minutos. 
dos esportes; regras básicas.

\section{Parte final:}

Volta à calma

Atividades de relaxamento e descontração.

\pm 5 minutos.

\section{População e amostra}

A população é constituída pelos 471 alunos de 11 a 17 anos matriculados no Instituto Educacional Vasconcelos Jardim no ano letivo de 2013. A amostra aleatória estratificada constante corresponde a 206 escolares. Foram selecionados 8 alunos por modalidade (turmas), sendo avaliados 112 escolares do sexo masculino e 94 do feminino.

\section{Instrumentos de coleta de dados}

Para a avaliação da AF, foi utilizado GPS da marca GARMIN, modelo 310XT (Validado por Firstbeat Technologies Ltda. Disponível em: < http://www.firstbeat.com >), com frequencímetro. Este GPS rastreia a posição com precisão e registra os dados com alta sensibilidade e precisão por meio de satélite HotFix. Para a avaliação do nível de AF foi utilizado o monitor de frequência cardíaca (FC) com uma unidade de interface para o processo de computação e um microcomputador. O sistema consiste em um leve transmissor fixado no tórax do indivíduo por um cinto que transmite os batimentos cardíacos a um relógio colocado no pulso do avaliado. É um método válido para monitorar FC em crianças ${ }^{11}$.

\section{Procedimento de coleta de dados}

Os sujeitos da pesquisa foram avaliados durante as aulas de Educação Física com duração de 50 minutos. No final de cada aula os dados da FC coletadas pelo monitor foram registrados no computador utilizando o sistema GARMIN CONNECT com Software de análise dos dados, transferidos online. Para analisar os resultados da monitorização da FC foi calculada a média dos batimentos cardíacos e a FC máxima atingida durante as aulas de Educação Física. A porcentagem de tempo em que a criança realizava AF nas diferentes faixas de FC foi definida conforme a proposta de pontos de corte sugerido por Armstrong $(1998)^{12}$ da seguinte forma: leve com FC abaixo $140 \mathrm{~b} / \mathrm{min}$, moderada entre 140 e $160 \mathrm{~b} / \mathrm{min}$ e vigorosa acima de $160 \mathrm{~b} / \mathrm{min}$.

A medida do desfecho (variável dependente), proporção média do tempo de aulas em AFMV foi obtida pela divisão do tempo médio das aulas despendido em AFMV pelo tempo de duração da aula.

A equipe de avaliadores foi composta por cinco professores de Educação Física, previamente treinados. Os GPSs foram ajustados e colocados em cada criança pelos professores de Educação Física no início das atividades em cada modalidade e retirado após o seu término. $\mathrm{Na}$ conclusão das atividades, foram arquivados os dados no sistema GARMIN CONNECT disponibilizados pelos equipamentos. Os GPSs foram programados para obter as seguintes informações: a) início e término das coletas das informações (tempo de aula), b) tempo em movimento em cada aula; c) gasto energético em quilocalorias (kcal) dispendida durante a aula ${ }^{13}$; d) frequência cardíaca média e máxima (b/min); e e) Nível de intensidade da AF (leve, moderada e vigorosa) durante as aulas.

Foram avaliadas 72 aulas (períodos) sendo realizadas um total de 386 avaliações. Foram feitas avaliações em todas as modalidades esportivas. Ocorreram $32 \%$ de perdas de observações por GPS com frequencímetro devido à falha de sinal do equipamento ou à ausência do participante nas aulas de Educação Física durante o estudo. Ainda, foram excluídos do 
estudo aqueles estudantes que apresentaram alguma dificuldade, permanente ou temporária, que o impossibilitasse de realizar alguma das atividades programadas pelos professores.

\section{Tratamento estatístico dos dados.}

Para a análise do perfil dos níveis de AF nas diferentes categorias de idade e sexo, inicialmente analisou-se os dados através de um estudo exploratório com o objetivo de avaliar os pressupostos essenciais da análise paramétrica. Para testar a normalidade das distribuições adotamos o teste de Shapiro-Wilks e a homogeneidade das variâncias o teste de Levene. Após, realizou-se a inspeção dos gráficos boxplot para a identificação de possíveis outliers realizada em todas as variáveis do estudo, nas diferentes categorias de idades e sexo. Os indivíduos identificados como outliers severos foram retirados da amostra.

Para a apresentação dos resultados utilizamos estatística descritiva através do cálculo de médias e desvios padrão para identificar a proporção média de tempo em AF nos diferentes níveis de intensidade, tempo de deslocamento ativo e gasto energético.

Para a identificação do tempo (minutos) e percentual de tempo em que escolares que realizaram AF nas intensidades leve, moderada e vigorosa, durante as aulas de Educação Física, utilizamos regra de três simples.

No que se refere as diferenças entre os sexos relacionado à AF, utilizou-se o teste " $t$ " de student para amostras independentes e a ANOVA seguida de post hoc de Bonferroni para identificar as possíveis diferenças entre as categorias de idades. Para verificarmos a possível influência do peso no dispêndio energético, utilizamos a Análise de Covariância (ANCOVA).

Para todas as análises estatísticas utilizamos o programa SPSS for Windows 20.0. O valor de significância previamente estabelecido para análise dos dados foi de $5 \%$.

\section{Procedimentos éticos}

Este estudo foi aprovado pelo Comitê de Ética em Pesquisa da Universidade Federal do Rio Grande do Sul - UFRGS. Processo n 2007719.

\section{Resultados}

\section{Nível de AF nas modalidades esportivas desenvolvidas na escola}

Com o intuito de identificar o comportamento da AF dos escolares nas diferentes modalidades esportivas, os resultados apontam desempenhos diferentes, tanto nas modalidades quanto ao sexo. No gráfico 1, apresentamos os resultados para o sexo masculino, onde se observa que: (1) as modalidades de Ciclismo e Futebol apresentam valores superiores em relação às outras no tocante a realização de atividades realizadas de forma vigorosa; (2) o Atletismo e o Voleibol são os esportes com a proporção média de tempo inferior (3) quando realizamos o somatório entre as AF realizada de forma moderada e vigorosa, é importante ressaltar que as modalidades de Ciclismo e Futebol de campo apresentam uma proporção média superior a $70 \%$ e as modalidades de Atletismo e Voleibol a proporção média cai para $50 \%$ do tempo total de aula nestas intensidades. 
Gráfico 1. Proporção média de tempo (min) dos rapazes nas diferentes intensidades de AF estratificado por modalidade esportiva.

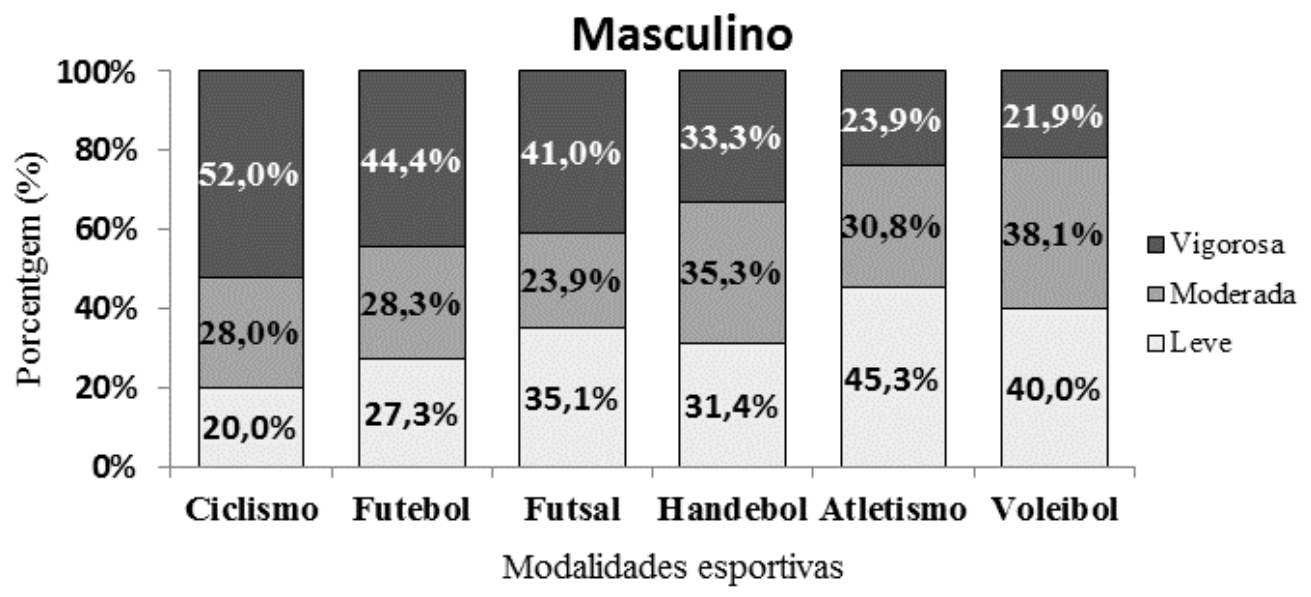

No tocante aos resultados das moças, no gráfico 2, apresentamos a proporção média de tempo em que escolares realizaram AF de forma vigorosa: (1) as modalidades de Ciclismo e Handebol apresentam valores superiores em relação às demais analisadas e; (2) as modalidades que apresentaram a menor proporção foram Caminhada Orientada e Voleibol. (3) no somatório entre as AF realizada de forma vigorosa e moderada, é importante ressaltar que as modalidades de Ciclismo, Handebol, Futsal e Caminhada foram as que apresentaram maior proporção média de tempo em AFMV, valores superiores a 60\%; já as modalidades de Atletismo e Voleibol apresentaram porcentagens inferiores, cerca de 50\% do tempo total das aulas de educação física são realizadas nestas faixas de intensidade.

Gráfico 2. Proporção média de tempo (min) das moças nas diferentes intensidades de AF estratificado por modalidade esportiva.

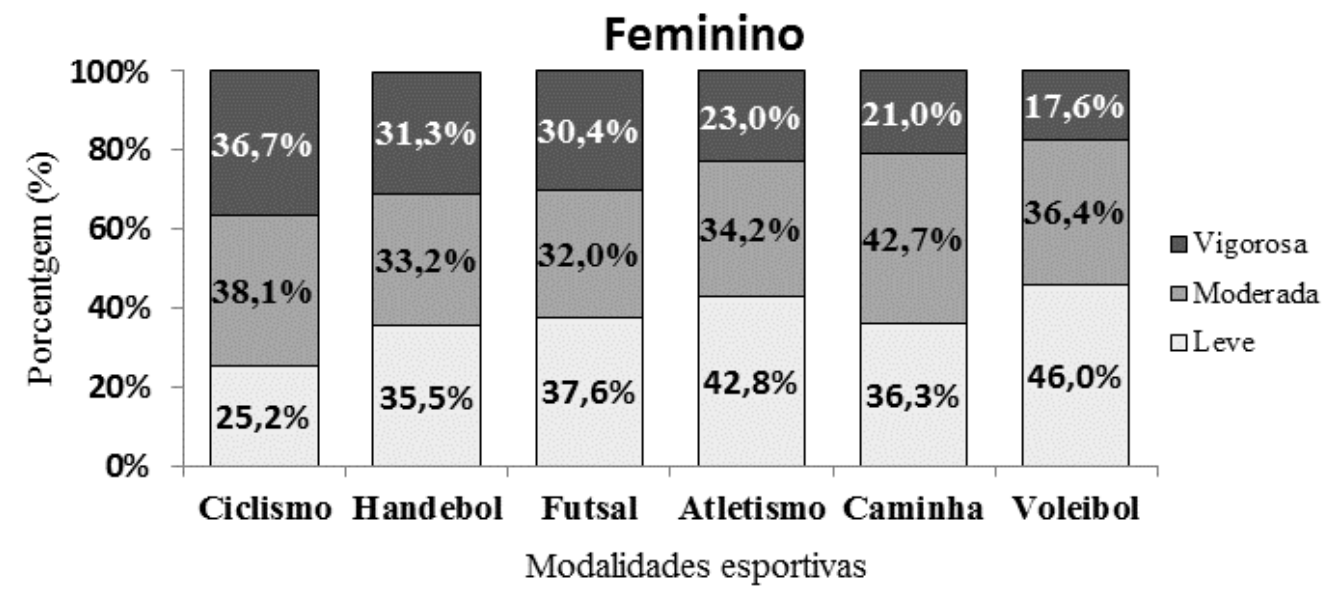

Outra variável importante deste estudo é o tempo de deslocamento ativo realizado durante as aulas de educação física na escola. Podemos observar na tabela 2 que as modalidades de Ciclismo e Caminhada orientada são as modalidades que apresentam valores próximos a $75 \%$ do tempo total de aula em movimento. Em seguida, as modalidades de Futebol e Futsal apresentam valores próximos de $60 \%$ e, Handebol e Voleibol, em torno de 50\%. Nesta variável a modalidade que apresentou valores inferiores foi atletismo, somente $40 \%$ do tempo de aula em deslocamento ativo. Ao compararmos os valores médios do tempo de deslocamento 
ativo entre rapazes e moças encontramos diferenças significativas $(p<0,05)$ nas modalidades de Futsal e Handebol, tendo os rapazes valores superiores em relação às moças (Tabela 2).

No que se refere aos resultados do gasto calórico (kcal) nas diferentes modalidades esportivas observamos que Ciclismo, Futebol e Futsal apresentaram valores superiores em relação às demais modalidades. Nestas modalidades a demanda energética ficou em torno de 250 kcal/aula. As modalidades de Handebol, Atletismo, Voleibol e Caminhada Orientada apresentaram valores inferiores, cerca de $200 \mathrm{kcal}$. Conforme apresentado na tabela 2, não há diferenças significativas entre rapazes e moças quanto ao gasto calórico nas aulas de educação física.

Tabela 2. Análise comparativa do tempo de deslocamento e gasto calórico de rapazes e moças nas modalidades esportivas.

\begin{tabular}{ccccc}
\hline \multirow{2}{*}{ TDA/aula } & Masculino & Feminino & & \\
\cline { 2 - 3 } & Média $\pm \mathbf{d p}$ & Média $\pm \mathbf{d p}$ & $\boldsymbol{t}$-valor & $\boldsymbol{p}$-valor \\
\hline Atletismo & $20: 34 \pm 02: 08$ & $20: 11 \pm 03: 12$ & 0,522 & 0,603 \\
\hline Caminhada & - & $38: 19 \pm 04: 05$ & - & - \\
\hline Ciclismo & $36: 01 \pm 04: 37$ & $39: 09 \pm 05: 21$ & $-1,568$ & 0,126 \\
\hline Futebol & $30: 38 \pm 03: 18$ & - & - & - \\
\hline Futsal & $30: 59 \pm 04: 25$ & $26: 21 \pm 05: 19$ & 3,288 & $0,002^{*}$ \\
\hline Handebol & $28: 20 \pm 04: 49$ & $25: 22 \pm 03: 37$ & 2,129 & $0,040^{*}$ \\
\hline Voleibol & $26: 16 \pm 03: 21$ & $25: 04 \pm 04: 12$ & 1,398 & 0,166 \\
\hline Kcal/aula & Média $\pm \mathbf{d p}$ & Média $\pm \mathbf{d p}$ & $\boldsymbol{t}-$ valor & $\boldsymbol{p}-$ valor \\
\hline Atletismo & $217 \pm 55,250$ & $205 \pm 39,107$ & 1,057 & 0,294 \\
\hline Caminhada & - & $178 \pm 40,218$ & - & - \\
\hline Ciclismo & $269 \pm 66,320$ & $248 \pm 43,239$ & 0,590 & 0,562 \\
\hline Futebol & $253 \pm 49,970$ & - & - & - \\
\hline Futsal & $246 \pm 60,876$ & $215 \pm 65,264$ & 1,590 & 0,117 \\
\hline Handebol & $210 \pm 51,045$ & $221 \pm 49,112$ & $-0,552$ & 0,587 \\
\hline Voleibol & $205 \pm 51,559$ & $213 \pm 49,008$ & $-0,740$ & 0,462 \\
\hline
\end{tabular}

Legenda: TDA = Tempo de deslocamento ativo em minutos; Kcal = Gasto energético em quilocalorias; dp. $=$ desvio padrão. $*$ = diferenças estatisticamente significativas $(\mathrm{p}<0,05)$.

Os resultados sugerem que as modalidades esportivas, embora tivessem a mesma estrutura no desenvolvimento das aulas (plano de aula), apresentam diferenças quanto ao comportamento da $\mathrm{AF}$, tempo de deslocamento ativo e gasto calórico. Provavelmente, devido às especificidades inerentes ao tipo de modalidade esportiva, infraestrutura e características das mesmas.

Resultados encontrados indicam que a prática de algumas modalidades esportivas possa exigir mais do que outras devido às características de cada uma delas. Nos dois sexos o voleibol foi a modalidade esportiva que menos exigiu dos escolares quanto ao nível de AF vigorosa, ao passo que as exigências maiores foram encontradas quando da prática do ciclismo, futebol de campo e futsal para o sexo masculino e, para o sexo feminino ciclismo, handebol e futsal.

\section{Níveis de intensidade, duração e gasto energético na AF realizadas durante a semana nas aulas de educação física}

Este tópico apresenta os resultados da proporção média de tempo de prática de AF nos diferentes níveis de intensidade, duração e frequência das aulas, bem como o gasto energético 
de acordo com a quantidade de modalidades esportivas frequentadas durante a semana conforme a proposta para a educação física escolar.

Inicialmente, apresentamos na tabela 3, a ocorrência do número de períodos semanais frequentadas pelos escolares nas aulas de educação física conforme a suas opções. Podemos identificar que os rapazes apresentam maior ocorrência de prática de AF, tendo os meninos uma associação na realização com quatro e seis períodos por semana. Já as moças, apresentam uma ocorrência inferior na prática de AF tendo uma associação com realização de somente quatro períodos semanais $(\mathrm{p}<0,05)$, ou seja, o mínimo obrigatório pela proposta. Os rapazes apresentam uma ocorrência maior em relação às moças quanto ao número de modalidades esportivas frequentadas na escola.

No que se refere à prática de AF realizada durante a semana, a tabela 4 , apresenta a média de tempo dos escolares nos diferentes níveis de intensidade, estratificada por categoria de idade e sexo.

Tabela 3. Frequência de ocorrência da quantidade de modalidades frequentadas durante a semana na escola.

\begin{tabular}{lccc}
\hline Modalidade/períodos & Masculino & Feminino (\%) & Total \\
\hline Duas - 4 períodos & $42,7 \% *$ & $64,0 \% *$ & $52,3 \%$ \\
\hline Três - 6 períodos & $34,5 \% *$ & $25,8 \%$ & $30,6 \%$ \\
\hline Quatro - 8 períodos & $15,5 \%$ & $9,0 \%$ & $12,6 \%$ \\
\hline Cinco - 10 períodos & $6,4 \%$ & $1,2 \%$ & $4,0 \%$ \\
\hline Seis - 12 períodos & $0,9 \%$ & $0,0 \%$ & $0,5 \%$ \\
\hline Total: & $100,0 \%$ & $100,0 \%$ & $100,0 \%$ \\
\hline
\end{tabular}

* Associação significativa entre as variáveis ( $\mathrm{p}<0,05)$.

Quanto ao tempo semanal engajado em práticas de AF realizadas de forma moderada a vigorosa, podemos observar na tabela 4, que o somatório de AFMV praticadas nas modalidades na escola demonstra valores significativos. Somente com as aulas de educação física os rapazes $(\mathrm{G} 1=187: 38 \pm 64: 18 ; \mathrm{G} 2=202: 57 \pm 69: 28 ; \mathrm{G} 3=175: 54 \pm 64: 24)$ cumprem com cerca de $2 / 3$ da carga horária semanal recomendada ${ }^{1}$ e, as moças $(\mathrm{G} 1=154: 19 \pm 51: 23$; G2 = 145:16 $\pm 38: 35 ; \mathrm{G} 3=136: 38 \pm 46: 43$ ), com valores inferiores ao apresentado pelos rapazes, cumprem com cerca de $50 \%$ da carga horária sugerida. Os resultados sugerem diferenças significativas $(\mathrm{p}<0,05)$ entre rapazes e moças nas três categorias de idade para AFMV.

Tabela 4. Análise comparativa do nível de AF (minutos), de rapazes e moças, realizada na semana, estratificado por categorias de idade e sexo. 


\begin{tabular}{|c|c|c|c|c|c|}
\hline \multirow[b]{2}{*}{ Categoria } & \multirow[b]{2}{*}{ NI } & Rapazes & Moças & \multirow[b]{2}{*}{$t$ - valor } & \multirow[b]{2}{*}{$p$ - valor } \\
\hline & & Média dp. & Média dp. & & \\
\hline \multirow{4}{*}{$\begin{array}{c}\text { C1 } \\
(11-13)\end{array}$} & AFL & $95: 24 \pm 34: 18$ & $61: 50 \pm 29: 23$ & 4,910 & $0,000^{*}$ \\
\hline & AFM. & $86: 37 \pm 31: 08$ & $95: 32 \pm 28: 13$ & $-1,400$ & 0,165 \\
\hline & AFV. & $101: 01 \pm 33: 18$ & $58: 47 \pm 23: 10$ & 6,905 & $0,000^{*}$ \\
\hline & Total: & $283: 02 \pm 93: 28$ & $216: 09 \pm 79: 00$ & 3,613 & $0,001 *$ \\
\hline \multirow{4}{*}{$\begin{array}{c}\mathrm{C} 2 \\
(14-15)\end{array}$} & AFL & $103: 47 \pm 0: 35: 43$ & $57: 03 \pm 20: 51$ & 6,587 & $0,000^{*}$ \\
\hline & AFM. & $93: 32 \pm 32: 59$ & $88: 20 \pm 20: 15$ & 0,784 & 0,436 \\
\hline & AFV. & $109: 25 \pm 36: 29$ & $55: 56 \pm 18: 20$ & 7,585 & $0,000^{*}$ \\
\hline & Total: & $306: 45 \pm 99: 29$ & $201: 19 \pm 56: 57$ & 5,357 & $0,000 *$ \\
\hline \multirow{4}{*}{$\begin{array}{c}\text { C3 } \\
(16-17)\end{array}$} & AFL & $89: 09 \pm 30: 02$ & $56: 56 \pm 28: 42$ & 3,203 & $0,003^{*}$ \\
\hline & AFM. & $79: 42 \pm 27: 36$ & $82: 54 \pm 28: 24$ & $-0,337$ & 0,738 \\
\hline & AFV. & $96: 12 \pm 36: 48$ & $52: 44 \pm 18: 19$ & 3,998 & $0,000^{*}$ \\
\hline & Total: & $265: 03 \pm 89: 11$ & $192: 34 \pm 74: 41$ & 2,518 & $0,016 *$ \\
\hline
\end{tabular}

Legenda: $\mathrm{NI}=$ Nível de intensidade; AFL = Atividade física leve; AFM = Atividade física moderada; $\mathrm{AFMV}=$ Somatório da Atividade física moderada e vigorosa; $\mathrm{AFV}=$ Atividade física vigorosa; dp. $=$ desvio padrão; $t=$ teste $t$ de Student para amostras independentes; $p=$ valor de significância $(p$ $=5 \%) ; *$ diferenças estatisticamente significativas $(\mathrm{p}<0,05)$;

Ao compararmos a proporção média de tempo de rapazes e moças nos diferentes níveis de intensidade e nas categorias de idade, observamos que os meninos apresentam valores superiores ao das meninas, havendo diferenças significativas nas intensidades leve e vigorosa nas três categorias de idade $(\mathrm{p}<0,05)$. Os rapazes apresentam o dobro de tempo engajado em práticas de $\mathrm{AF}$ realizadas de forma vigorosa em relação àquelas. Além disso, os rapazes apresentam a proporção média de tempo crescente da categoria $\mathrm{C} 1$ para o $\mathrm{C} 2 \mathrm{e}$, uma estabilização do tempo no grupo 3. Inversamente, as meninas apresentam valores decrescentes com passar do tempo, o que ilustra certa preocupação.

No tocante ao somatório do tempo de deslocamento ativo na semana, podemos observar na tabela 5, que a proporção média dos rapazes em movimento é superior ao das moças em todas as categorias de idade $(\mathrm{p}<0,05)$. Quando analisamos as diferenças entre as categorias de idade esta variável não apresentou diferenças significativas ao longo do tempo $(p>0,05)$ tanto para rapazes quanto para moças.

Tabela 5. Análise comparativa do tempo de deslocamento e gasto energético realizado durante a semana nas aulas de Educação Física, estratificada por categorias e idade e sexo.

\begin{tabular}{|c|c|c|c|c|c|}
\hline & & Rapazes & Moças & & \\
\hline $\mathbf{V}$ & Ca 1 & Média $\pm d p$ & Média $\pm d p$ & $t$ - valor & $p$ - valor \\
\hline \multirow{2}{*}{ TDA/aula $^{\mathrm{a}}$} & $\mathrm{C} 1(1$ & $168: 30$ &  & 2,974 & $0,004 *$ \\
\hline & C2 (14-15) & $178: 52 \pm 58: 50$ & $122: 50 \pm 24: 43$ & 4,216 & $0,000 *$ \\
\hline
\end{tabular}




\begin{tabular}{llllll}
\hline & $\mathrm{C} 3(16-17)$ & $160: 46 \pm 55: 12$ & $113: 22 \pm 34: 52$ & 2,697 & $0,011^{*}$ \\
\hline \multirow{3}{*}{ Kcal/aula } & $\mathrm{C} 1(11-13)$ & $1315 \pm 427,931$ & $1012 \pm 330,489$ & 3,705 & $0,000^{*}$ \\
\cline { 2 - 7 } & $\mathrm{C} 2(14-15)$ & $1423 \pm 457,242$ & $953 \pm 244,149$ & 5,272 & $0,000^{*}$ \\
\cline { 2 - 7 } & $\mathrm{C} 3(16-17)$ & $1237 \pm 429,835$ & $921 \pm 289,850$ & 2,383 & $0,022^{*}$ \\
\hline
\end{tabular}

Legenda: $\mathrm{Kcal}=$ Gasto energético em quilocalorias; TDA $=$ Tempo de deslocamento ativo em minutos; dp. $=$ desvio padrão ${ }^{*}=$ diferenças estatisticamente significativas $(\mathrm{p}<0,05) ;{ }^{\mathrm{a}}=$ Modalidades de ciclismo e caminhada orientada não foram computados.

No que se refere à comparação dos resultados do gasto energético (Kcal), de rapazes e moças, dependidos nas aulas de educação física durante a semana, retiramos o efeito da variável massa corporal $(\mathrm{Kg})$ com o intuito de identificar se a mesma poderia influenciar no desenvolvimento do comportamento desta variável no tempo. Feito isso, os resultados sugerem um comportamento díspar em relação ao sexo (Tabela 5). Ao analisarmos os resultados nas diferentes categorias de idade, observamos que os escolares do sexo masculino apresentam demanda energética superior em relação às moças nas três categorias de idade $(\mathrm{p}<0,05)$. Os rapazes apresentam um incremento na quantidade de kcal gastas com o passar do tempo demonstrando diferenças significativas $(\mathrm{p}<0,05)$ entre os grupos analisados, enquanto as moças apresentam uma estabilização da quantidade de quilocalorias gastas ao longo do tempo não sendo observadas diferenças.

Quanto à contribuição da educação física escolar no alcance das recomendações dos 300 minutos semanais de AF realizadas de forma moderada a vigorosa, averiguamos contribuições significativas nos níveis de AF apresentado pelo presente programa de intervenção. Uma explicação plausível para esta contribuição nas recomendações da AF é de que as características do programa favorecem os escolares na perspectiva de eleger quais e quantas modalidades esportivas desejam frequentar, bem como os horários conforme seus interesses.

Outro aspecto que convém ressaltar é que rapazes e moças possuem preferências diferentes quanto às opções pelas práticas esportivas realizadas na escola; as moças apresentam uma associação com as modalidades de voleibol e caminhada orientada e os rapazes com as modalidades de ciclismo, futebol e futsal. Assim, a magnitude das diferenças aumenta de forma significativa a favor dos rapazes quando calculamos os níveis de AF semanal. Os resultados demonstram que, além de frequentar mais modalidades do que as moças, os rapazes optam por modalidades esportivas que apresentam exigências físicas superiores.

\section{Discussão}

Os resultados desta pesquisa expõem um comportamento diferente quanto as preferências dos escolares em relação as práticas corporais escolhidas e realizadas na escola. Os rapazes optam em realizar atividades como Ciclismo, Futebol e Futsal enquanto as meninas Vôlei e Caminhada Orientada.

Não obstante, mesmo tendo uma estrutura de aula semelhante, as modalidades esportivas apresentam características e comportamentos distintos quanto ao nível de intensidade e duração da prática de AF, geralmente as modalidades de preferência dos rapazes são as que exibem comportamentos mais ativos, enquanto as moças apresentam desempenhos menos ativo.

Nos estudos realizados por Guedes e Guedes ${ }^{14,15}$ os autores apresentam resultados semelhantes ao exposto pela presente investigação, as preferências esportivas são diferentes entre rapazes e moças. Nos dois estudos, realizados com uma amostra aleatória, avaliando a frequência cardíaca de escolares nas aulas, demonstraram que os batimentos cardíacos elevam mais em algumas modalidades esportivas do que outras, ilustrando as mesmas variações ao 
apresentado pela presente investigação, sendo o Futebol e Futsal com valores superiores ao apresentado pela modalidade de Voleibol.

Nesta perspectiva, alguns programas diferenciados para a educação física escolar têm sido desenvolvidos com a missão de ampliar a quantidade e qualidade das práticas corporais realizadas na escola. Deste modo, Matsudo et al. (2003) ${ }^{16}$ apresentaram os resultados da comparação dos níveis de aptidão física entre duas propostas diferenciadas entre escolas privadas (proposta diferenciada) e escolas públicas (modelo convencional), os resultados sugerem que rapazes e moças das escolas privadas submetidos a um programa de intervenção diferenciado foram superiores quanto ao nível de aptidão física relacionada à saúde.

Outro estudo que demonstrou resultados satisfatórios foi o de Ribeiro (2009) ${ }^{7}$, que analisou o efeito de dois programas de intervenção no nível de AF de adolescentes da rede pública de São Paulo/SP, durante 4 meses. Os resultados apontam que após o período de intervenção houve um aumento considerável da prática total de AF semanal.

$\mathrm{Na}$ mesma linha de investigação, Barros et al. (2009) ${ }^{17}$ analisaram os resultados do Projeto Saúde na Boa, realizado em Recife e Florianópolis, divididos em dois grupos: a) Grupo de intervenção; b) Grupo controle. Para o grupo intervenção os dados demonstram um aumento dos escolares considerados ativos após a implantação e realização das atividades, enquanto para o grupo controle, os resultados apontam um aumentou na quantidade de indivíduos considerados inativos.

Beets et al. (2005) ${ }^{18}$ realizaram um estudo comparando as variáveis da aptidão física relacionada à saúde de escolares que participavam de educação física e esportes realizadas uma, duas ou mais de três modalidades esportivas frequentadas durante a semana, comparados com estudantes que participavam unicamente de educação física na escola. Os resultados demonstram que quanto maior o número de práticas de atividades físicas maiores eram os níveis de aptidão física quando relacionado com as crianças que frequentavam somente as aulas no modelo tradicional ( 2 períodos semanais de 50 minutos).

Outra pesquisa que apresenta resultados favoráveis aos programas diferenciados para a educação física foi o apresentado por Pate et al. (2009) ${ }^{19}$. Com uma proposta auxiliar observaram um aumento dos níveis de AF durante as aulas de Educação Física. Os resultados encontrados neste estudo demonstraram que as crianças apresentavam 37\% do tempo em AFMV, no entanto, quando foram submetidas a um programa de intervenção, a proporção subiu para $51 \%$.

Com resultados satisfatórios Thompson et al. $(2013)^{20}$, em um estudo realizado nos distritos escolares da Califórnia (San Fracisco Unified School - SFUSD) apresenta a quantidade de períodos semanais para educação física escolar, cerca de 100 minutos para o nível fundamental séries iniciais e 200 minutos para os níveis fundamental séries finais e ensino médio, o dobro ao apresentado pela maioria das escolas em nossos país. Em outro estudo também realizado nos EUA, Nader $(2003)^{21}$, ao avaliar 814 crianças através do SOFIT observaram que $30,2 \%$ tinham uma aula por semana, $45,3 \%$ duas vezes por semana e $24,5 \%$ acima de três vezes por semana.

Quando analisamos o somatório do tempo de AF realizadas na semana, os resultados da presente investigação demonstram valores positivos nos dois sexos e nas diferentes categorias de idade, esta característica pode ser considerada como o diferencial desta proposta para educação física na escola, contribuindo significativamente com as necessidades de 300 minutos de práticas de AFMV por semana. Somente com as aulas de educação física os rapazes cumprem com cerca de $2 / 3$ da carga horária semanal recomendada de 300 minutos e, as moças cerca de $50 \%$ da carga horária sugerida, diferentemente do apresentado por outros estudos para AF semanal ${ }^{2,17,22}$.

Assim sendo, a AF universalmente reconhecida como importante objetivo nos programas de educação física escolar infelizmente indica que os esforços físicos a que são expos- 
tos os escolares nas aulas de educação física no formato tradicional, não vêm repercutindo de maneira adequada para se alcançar níveis mais elevados de aptidão física relacionada à saú$\mathrm{de}^{10,15}$. Torna-se necessário refletirmos com as autoridades da área da educação, políticas públicas que garantam um número adequado de horas por semana, assim como as condições necessárias para as aulas de educação física na perspectiva de contemplar nossos escolares com conteúdo que tenham utilidade para sua vida, com práticas corporais satisfatórias e diversificadas e com a carga horária adequada para o desenvolvimento saudável de nossos estudantes.

Embora a presente proposta para educação física escolar enfatize a prática de esportes de maneira mais efetiva à aquisição de hábitos e atitudes que possam ser permanentes e mais aderentes, destacamos que a proposta também oferece atividades extraclasses como, ações educativas, seminários conjuntos com outras disciplinas, debates sobre os benefícios da prática de atividade física na promoção da saúde com o intuito de discutir com nossos alunos, pais, professores e funcionários sobre o papel da educação física e seus compromissos enquanto disciplina na escola.

\title{
Conclusão
}

Os resultados da presente investigação sugerem que a realização de um programa de atividades físicas, planejado, organizado focado nos interesses dos alunos, aumentará os níveis de AF dos escolares ao longo do tempo provendo os aspectos associados à saúde. Os níveis de intensidade e duração dos esforços físicos realizados alcançaram de forma satisfatória quanto ao nível necessário para a promoção da saúde, principalmente quando do somatório de tempo na semana.

Quanto ao tempo semanal engajado em práticas de AF realizadas de forma moderada a vigorosa, observamos que o somatório de AFMV praticadas nas modalidades na escola demonstram resultados significativos, somente com as aulas de educação física os rapazes cumprem com cerca de $2 / 3$ da carga horária semanal recomendada e, as moças com valores inferiores ao apresentado pelos rapazes, cumprem com cerca de $50 \%$ da carga horária sugerida.

Consideramos estes resultados o diferencial desta proposta, neste formato os escolares ampliam o tempo de prática de AF na escola contribuindo de forma significativa quando comparada com as recomendações de 300 minutos de AF moderada a vigorosa por semana.

\section{PHYSICAL ACTIVITY LEVELS IN DIFFERENT SPORTS: A NON- CONVENTIONAL PHYSICAL EDUCATION SCHOOL PROGRAM}

\begin{abstract}
Objectives: a) To assess intensity, displacement time and energy expenditure (Kcal) in different sports modalities, stratified by gender; b) To identify the contribution of school physical education in moderate and vigorous physical activity (MVPA) time according to the amount of sports modalities enrolled during the week. Methods: Case study: physical education program at Instituto Estadual de Educação Vasconcelos Jardim School, RS, Brazil. 206 schoolchildren (112 boys and 94 girls) were randomly selected and assessed at 72 classes using GPS GARMIN, 310XT model, with frequency meter. The intensity of physical activity (PA) was categorized according to Armostrong (1998) criteria. Descriptive statistics was used to identify time ratio in the different PA levels; T Student test was used for comparisons between genders and ANOVA with Bonferroni post hoc test to comparisons between ages. Results: The sum of MVPA practiced in sports modalities showed significat results: boys reached 2/3 of the recommend hours per week in MVPA, while girls reached 50\%, considering only physical
\end{abstract}


education classes. We consider that these results are the difference of this proposal, since this format contributes significantly to reach the recommendations of 300 minutes of moderate to vigorous physical activity per week.

Keywords: Physical Activity. Intensity and Duration. Schoolchildren

\section{Referências}

1. World Health Organization. Inequalities Young people's health: key findings from the Heath Behaviour in School-aged Children survey fact sheet. Disponível em: www.euro.who.into/document/Mediacentre [10 de dezembro de 2010].

2. Halall PC, Bertoldi AD, Goncalves H, Victora CG. Prevalência de sedentarismo e fatores associados em adolescentes de 10-12 anos de idade. Cad Saúde Pública (online) 2006;22(6):1277-1287.

3. Silva DAS, Petroski EL, Pelegrini A. Da evidência à intervenção: programa de exercícios físicos para adolescentes com excesso de peso em Florianópolis, SC. Rev. bras. ativ. fís. Saúde 2009;14(2):139-146.

4. Guedes DP, Guedes JERP, Barbosa DS, Oliveira JA. Níveis de prática de atividade física habitual em adolescentes. Rev Bras Med Esporte 2001;7(6):187-199.

5. Cunha CT. Impacto do programa educativo no gasto energético de escolares nas aulas de educação física: ensaio randomizado controlado. São Paulo: Master Thesis, Universidade Federal de São Paulo, 2002.

6. Farias ES, Paula F, Carvalho WRG, Gonçalves EZ, Baldin AD, Guerra-Júnior G. Efeito da atividade física programada sobre a composição corporal em escolares adolescentes. J Pediatr. 2009;85(1):28-39.

7. Ribeiro EHC, Florindo AA. Efeitos de um programa de intervenção no nível de atividade física de adolescentes de escolas públicas de uma região de baixo nível socioeconômico: descrição dos métodos utilizados. Rev. bras. ativ. fís. Saúde. 2010;15:28-34.

8. Hallal PC, Dumith SC, Bastos JP, Reichert FF, Siqueira FV, Azevedo MRJ. Evolução da pesquisa epidemiológica em atividade física no Brasil: revisão sistemática. Rev. Saúde Públ. 2007;41(3):453-460.

9. Guedes DP, Souza MV, Ferreirinha JE, Silva AJRM. Physical activity and determinants of sedentary behavior in brazilian adolescents from an underdeveloped region. Perceptual and Motor Skills 2012;114(1):542-552.

10. Gaya ACA, Lemos AT, Gaya A, Teixeira D, Pinheiro E, Moreira RB. Manual de aplicação de medidas e testes, normas e critérios de avaliação. Projeto Esporte Brasil (PROESP-Br). 2012. Disponível em: http://www.proesp.ufrgs.br [20 de dezembro de 2012].

11. Armstrong N, Bray S. Physical activity patterns defined by continuous heart rate monitoring. Archives of Diasease in Chilhood 1991;66(1):245-247. 
12. Armstrong N. Yuong people's physical activity patterns as assessed by heart rate monitoring. Journal of sports Sciences 1998;16(1):9-16.

13. Firstbeat Technologies. An Energy Expenditure Estimation Method based on Heart Rate Measurement. Firstbeat Technologies Ltda, 2007. Disponível em: http://www.firstbeat.com/consumers/firstbeat-intelligence-in-heart-ratemonitors\#energyexpenditure.html_[20 de outubro de 2013].

14. Guedes JERP, Guedes DP. Características dos programas de educação física escolar. Rev paul. Educ. Fís 1997;11(1):46-62.

15. Guedes JERP, Guedes DP. Esforços físicos nos programas de educação física escolar. Rev paul. Educ. Fís 2001;15(1):33-44.

16. Matsudo VKR, Andrade DR, Matsudo SMM, Araújo FL, Andrade E, Braggion G, Ribeiro MA. Construindo saúde por meio da atividade física em escolares. R. Bras. Ci. e Mov. 2003;11(4):111-118.

17. Barros MV, Nahas MV, Hallal PC, et al. Effectiveness of a School-Based Intervention on Physical Activity of High School Students in Brazil: The Saúde na Boa Project. Journal of Physical Activity and Health 2009;6(1):163-169.

18. Beets MW, Pitetti KH. Contribuition of Physical Education and Sports to Health-Related Fitness in High School students. Journal of School Health 2005;75(1):25-30.

19. Pate RR, Stevens J, Webber LS, et al. Age-related change in physical activity in adolescents girls. J Adolesc Health 2009;44(3):275-82.

20. Thompson HR, Linchey J, Madsen AK. Are Physical Education Policies Working? A Snapshot from San Francisco, 2011. Preventing Chornic Disease 2013;10(1):1-10.

21. Nader PR. National Institute of Child Health and Human Development Study of Early Child Care and Yoult Development Network. Frequency and intensity of activity of thirdgrade children in physical education. Arch Pediatr Adolesc Med. 2003;157(2):185-190.

22. Silva DAS, Lima JO, Silva RJS, Prado RL. Nível de atividade física e comportamento sedentário em escolares. Rev Bras Cineantropom Desempenho Hum 2009;11(3):299-306.

Recebido em: 21/08/2015

Revisado em: 23/11/2015

Aprovado em: 25/08/2016

Endereço para correspondência:

rbmoreira2@gmail.com

Rodrigo Baptista Moreira

Universidade Luterana do Brasil

Rua Borges de Medeiros, ${ }^{\circ} 259$

General Câmara, RS, CEP: 95820-000 